\title{
An algorithm computing homotopy groups
}

\author{
Pedro Real ${ }^{1}$ \\ Dpto. Matemática Aplicada I, Fac. de Inform. Y Estadistia, Univ. Sevilla, c/Tarfia s/n, 41012 Sevilla, Spain
}

\begin{abstract}
An algorithm computing homotopy groups of a reduced simplicial set with effective homology is described, using the Whitehead tower method.
\end{abstract}

\section{Introduction}

The effective homology theory [6,7] is a powerful and convenient tool to design algorithms computing the various invariants (homology groups, homotopy groups, etc.) of algebraic topology.

An algorithm computing homotopy groups, via the Whitehead tower, is described in this paper. As usual this theoretical solution ignores practical time and storage restrictions, but the results already obtained by similar methods for homology groups of iterated loop spaces [5] show that in this way we should be able to actually reach new homotopy groups for a significant variety of cases. In Section 2, the main mathematical objects of the problem are defined. In Section 3, the effective homology version of the Serre spectral sequence is described. Section 4 is devoted to Eilenberg-MacLane spaces, an essential tool in the Whitehead tower process. Finally, in Section 5, these results are put together to obtain the algorithm computing homotopy groups.

\section{Simplicial sets and effective homology}

A combinatorial model is needed to represent the topological spaces we are interested in. The usual tool is the notion of simplicial set. A simplicial set is a collection of simplices, each having a dimension (a positive integer), as also a collection of face operators, and a collection of degeneracy operators [3]. For example, a (solid) triangle with three vertices, three edges and one triangle can be described as a simplicial set $T=\left\{T_{n}, \partial_{i}^{n}, \eta_{i}^{n}\right\}$, where

- The set of $n$-simplices $T_{n}$ is the set of (weakly) increasing $(n+1)$-sequences $\left(a_{0}, \ldots, a_{n}\right)$ where $0 \leqslant$ $a_{0} \leqslant \cdots \leqslant a_{n} \leqslant 2$

\footnotetext{
I Tel.: 1934 54552794; fax: 1934 54557878; e-mail: real@ obelix.cica.es.
} 
- the $i$ th face $\partial_{i}^{n} \sigma$ of the $n$-simplex $\sigma=\left(a_{0}, \ldots, a_{n}\right)$ is simply obtained by removing the $i$ th vertex: $\partial_{i}^{n}(\sigma)=\left(a_{0}, \ldots, a_{i-1}, a_{i+1}, \ldots, a_{n}\right)$;

- on the contrary, the $i$ th degeneracy of the same simplex is obtained by duplicating the $i$ th vertex: $\eta_{i}^{n}(\sigma)=$ $\left(a_{0}, \ldots, a_{i}, a_{i}, \ldots, a_{n}\right)$.

A simplex is non-degenerate if it is not the image of some simplex by a degeneracy operator. In the example above, the simplex $\sigma=\left(a_{0}, \ldots, a_{n}\right)$ is non-degenerate if and only if the sequence $\sigma$ is strictly increasing; so that there are only three non-degenerate 0 -simplices (three vertices), three non-degenerate 1 -simplices (three edges), and one non-degenerate simple (the triangle). The same example can be given with any positive integer $d$ instead of 2 . In the geometric realization of a simplicial set (see [3]), only the non-degenerate simplices contribute.

The chain complex $C_{*} K$ associated with a simplicial set $K$ is a differential $Z$-graded module where the $n$-component is the free $Z$-module $C_{n} K=Z\left[K_{n}\right]$ generated by the $n$-simplices of $K$, and the boundary operator $d_{n}: C_{n} K \rightarrow C_{n-1} K$ is the alternate sum $d_{n}=\Sigma_{0 \leqslant i \leqslant n}(-1)^{i} \partial_{i}^{n}$. The homology groups $H_{*} K$ are the quotient groups $H_{n} K=\operatorname{ker} d_{n} / \mathrm{im} d_{n+1}$.

Let us consider, for example, the chain complex $C_{*} T$. Its homology contains only one $Z$ component in dimension 0 , the other components being null. This is more precisely described as follows. Let $H C_{*}$ be the chain complex with only a $Z$ component in dimension 0 , generated by "*". Then two chain complex morphisms can be defined, the first one $f: C_{*} T \rightarrow H C_{*}$, the second one $g: H C_{*} \rightarrow C_{*} T$ :

$$
\begin{array}{lll}
f_{0}: C_{0} T \rightarrow H C_{0}:(i) \longmapsto * ; & f_{n}=0 & \text { if } n>0 ; \\
g_{0}: H C_{0} \rightarrow C_{0} T: * \longmapsto(0) ; & g_{n}=0 & \text { if } n>0 .
\end{array}
$$

The composition $f \circ g$ is the identity morphism so that the small complex $H C *$ is a direct summand of the big complex $C_{*} T$. In fact $H C *$ describes the homology of $C_{*} T$ because the composition $g \circ f$ is homotopic to the identity; this means there exists a homotopy operator $h: C_{*} T \rightarrow C_{*} T$ of degree 1 satisfying the relation $d h+h d=1-g f$; the homotopy operator is defined as follows:

$$
h_{n}: C_{n} T \rightarrow C_{n+1} T:\left(a_{0}, \ldots, a_{n}\right) \longmapsto\left(0, a_{0}, \ldots, a_{n}\right) .
$$

A 5-uple $\left(C_{*} T, H C_{*}, f, g, h\right)$ is called a reduction or special homotopy equivalence. There is a simple generalization of this notion which is called a homotopy equivalence and a simplicial set with effective homology is a 4-uple $\left(K, C_{*} K, H C_{*}, \epsilon\right)$ where $K$ is a simplicial set, $C_{*} K$ is the chain complex canonically associated to $K, H C_{*}$ is a chain complex of finite type in each dimension and $\epsilon$ is a homotopy equivalence between $C_{*} K$ and $H C_{*}$. The homology groups of the small complex $H C_{*}$ can be computed and on the contrary the big chain complex $C_{*} K$ could for example not to be of finite type. But the homotopy equivalence $\epsilon$ proves the homology groups are the same.

Proposition 1. An algorithm homology-group can be implemented:

homology-group : $\left(X_{\mathrm{EH}}, n\right) \longmapsto H_{n}(X)$,

where $X_{\mathrm{EH}}$ is a simplicial set with effective homology and $H_{n}(X)$ is the nth homology group of the underlying simplicial set $X$.

The main result considered in this paper is the following theorem. A simplicial set is 1-reduced if it has only one vertex (0-simplex) and no non-degenerate 1 -simplex. 
Theorem 2. An algorithm homotopy-group can be implemented:

homotopy-group : $\left(X_{\mathrm{EH}}, n\right) \longmapsto \pi_{n}(X)$,

where $X_{\mathrm{EH}}$ is a 1-reduced simplicial set with effective homology and $\pi_{n}(X)$ is the nth homotopy group of the underlying simplicial set $X$.

This theorem was proved in 1956 by Brown [1], but Brown himself explained that his "algorithm" is without practical use, because of enormous space and time complexity. On the contrary, the algorithm roughly described here can certainly be applied in a significant number of cases, and a nice challenge of actual implementation is so opened.

\section{Effective homology of principal fibrations}

A fibration is a kind of twisted product. To define a fibration, several ingredients are needed: a base space $B$, a fiber space $F$, a structural group $G$ acting on the fiber, and finally a twisting operator $\tau$ which looks like a function $B \rightarrow G$ satisfying some coherence conditions. In particular, such a structure can be defined in the simplicial framework, see [4]. When these objects are given, the total space of the fibration is denoted $F \times{ }_{\tau} B$ and is essentially the product of $B$ by $G$ twisted according to the $\tau$-operator. For example a description of the 3-sphere as a twisted product of the 2-sphere by the 1-sphere (circle) can be given this way (Hopf fibration).

The key tool of the effective homology theory is that if two spaces among three ones, base space, fiber space and total space of a fibration, are simplicial sets with effective homology, then a description of the third one also as a simplicial set with effective homology can be deduced [7]. We will use two particular cases.

Theorem 3. An algorithm serre can be implemented:

$$
\text { serre: }\left(B_{\mathrm{EH}}, F_{\mathrm{EH}},(B, F, G, \tau)\right) \longmapsto E_{\mathrm{EH}} \text {, }
$$

where $(B, F, G, \tau)$ is a simplicial fibration, $B_{\mathrm{EH}}$ is a description of the base space as a simplicial set with effective homology, $F_{\mathrm{EH}}$ the same for the fiber space, and the result $E_{\mathrm{EH}}$ is an analogous description of the total space.

Theorem 4. An algorithm eilenberg-moore can be implemented:

$$
\text { eilenberg-moore : }\left(F_{\mathrm{EH}}, E_{\mathrm{EH}},(B, F, G, \tau)\right) \longmapsto B_{\mathrm{EH}} \text {, }
$$

where this time the datum $E_{\mathrm{EH}}$ is a description of the total space $B \times_{\tau} F$ as a simplicial set with effective homology.

The first algorithm is the effective homology version of the Serre spectral sequence; the same for the second and the Eilenberg-Moore spectral sequence. 


\section{Eilenberg-MacLane spaces}

If $G$ is a simplicial group, that is, a simplicial set provided with a group structure, compatible in a natural sense with the simplicial structure, then the classifying space $B G$ is defined. This space is the base space of a universal fibration whose structural group is $G$. Such a space $B G$ is well defined up to homotopy. If $E G$ is the total space of the universal fibration over $G$, then $E G$ is a contractible space and it is very easy to describe its effective homology. So if $G$ is also a simplicial group with effective homology, Theorem 4 allows to produce the effective homology of the classifying space $B G$.

Theorem 5. An algorithm classifying can be implemented:

$$
\text { classifying : } G_{\mathrm{EH}} \longmapsto B G_{\mathrm{EH}}
$$

where the underlying space $B G$ of the simplicial set with effective homology $B G_{\mathrm{EH}}$ is the classifying space of $G$, the underlying simplicial group of $G_{\mathrm{EH}}$.

Furthermore, if $G$ is a commutative group, then $B G$ is naturally provided with a commutative simplicial group structure, so that the classifying process can be iterated. In particular, if $\pi$ is an ordinary (discrete) abelian group, it can be considered as a simplicial group where the vertices are the elements of $\pi$ and without any other non-degenerate simplex. Then the simplicial group $K(\pi, n)$ is defined as follows: $K(\pi, 0):=\pi$ and $K(\pi, n+1)=B K(\pi, n)$. If $\pi$ is of finite type, then it is very simple to compute the effective homology of $K(\pi, 1)=B \pi$ : it is essentially the classical homology of the group $\pi$.

Theorem 6. An algorithm emleh (Eilenberg-MacLane effective homology) can be implemented:

$$
\text { emleh : } K(\pi, n) \longmapsto K(\pi, n)_{E H},
$$

where $\pi$ is an abelian group of finite type, $n$ is a positive integer, and the result is a version with effective homology of the nth Eilenberg-MacLane space $K(\pi, n)$ associated with $\pi$.

\section{The Whitehead tower process}

The Whitehead tower process is an iterative scheme which allows one to reach any homotopy group of a 1-reduced simplicial set. Let $E=E_{2}$ be a 1-reduced simplicial set; the index 2 explains that the homology and therefore the homotopy as well does not begin before the dimension 2; this is a consequence of the "1-reduced" hypothesis. The non-1-reduced problem has a quite different nature and in fact is undecidable (Novikov).

The Whitehead iteration begins at the step 2, and at the step $n$ starting from $E_{n}$ computes $\pi_{n}(E)$ and the next needed space $E_{n+1}$. The step $n$ of the Whitehead process works as follows:

(1) $E_{n}$ is a simplicial set with effective homology; its homology and homotopy does not begin before dimension $n$ and an algorithm computes $H_{n}\left(E_{n}\right)=\pi_{n}\left(E_{n}\right)$ (Hurewicz theorem); in fact $\pi_{n}\left(E_{n}\right)=$ $\pi_{n}(E)$ a group which was looked for;

(2) a canonical fibration $K(\pi, n-1) \rightarrow E_{n+1} \rightarrow E_{n}$ can be constructed where $\pi$ is the group $\pi_{n}\left(E_{n}\right)$ just computed, the fiber space is an Eilenberg-MacLane space. Because of the effective homology version 
of the Serre spectral sequence, and because $K(\pi, n-1)$ can be organized as a simplicial group with effective homology, an algorithm can construct $E_{n+1}$ as a simplicial set with effective homology.

This proves Theorem 2.

\section{Concluding remarks}

Two main challenges are opened by these results. The first one consists in implementing the algorithms here roughly described and to see to what extent they are able to compute homotopy groups. Of course there is no hope to compute in this way, for example, the new stable sphere homotopy groups; for the last groups, very specific, deep and powerful methods have been designed which of course cannot be overshadowed by the general method explained here. But for a general space, our method should be of significant interest.

Furthermore Henri Cartan discovered very efficient methods to compute the usual homology groups of Eilenberg-MacLane spaces [2]. Of course it is possible to use these methods to significantly improve the computation of the effective homology of those spaces, sketched above.

A large and fascinating working field is so opened.

\section{References}

[1] E.H. Brown Jr., Finite computability of Postnikov complexes, Ann. of Math. 65 (1957) 1-20.

[2] H. Cartan, Algèbres d'Eilenberg-MacLane, in: Euvres (Springer, Berlin, 1979).

[3] P. Gabriel and M. Zisman, Calculus of fractions and homotopy theory, Ergebnisse der Mathematik, 35 (Springer, Berlin, 1967).

[4] D.M. Kan, A combinatorial definition of homotopy groups, Comment. Math. Helv. 67 (1958) 282-312.

[5] J. Kubio and E. Sergeraert, A program computing the homology groups of loop spaces, SIGSAM Bulletin 25 (1991) $20-24$.

[6] F. Sergeraert, Homologic effective, Comptes-rendus hebdomadaires des séances de l'Académie des Sciences, Paris, Série A, 304 (1987) 279-282; 319-321.

[7] F. Sergeraert, The computability problem in algebraic topology, Adv. in Math., To appear. 